\title{
Peer group-based online intervention program to empower families of those raising children with disabilities: Study protocol for a non-randomized waitlist-controlled trial
}

\author{
Akemi Matsuzawa \\ Ibaraki Christian University \\ Rie Wakimizu ( $\sim$ riewaki@md.tsukuba.ac.jp ) \\ University of Tsukuba https://orcid.org/0000-0003-2464-6028 \\ Iori Sato \\ University of Tokyo \\ Hiroshi Fujioka \\ Ibaraki Prefectural University of Health Sciences: Ibaraki Kenritsu Iryo Daigaku \\ Kaori Nishigaki \\ St. Luke's International University \\ Seigo Suzuki \\ Tokyo Medical University \\ Naoko Iwata \\ University of Tsukuba, Tsukuba hospital
}

\section{Study Protocol}

Keywords: disabled children, family, family empowerment, caregiver burden, health care service, QOL, peer group, online program, non-randomized waitlist-controlled trial

Posted Date: October 29th, 2021

DOI: https://doi.org/10.21203/rs.3.rs-998023/v1

License: (c) (i) This work is licensed under a Creative Commons Attribution 4.0 International License. Read Full License 


\section{Abstract}

\section{Background}

Families raising children with disabilities assume risks to their health and lives. Therefore, it is necessary to support these families to improve family empowerment, which is the ability of these families to control their own lives and to promote the collaborative raising of children with disabilities. This is the first online program development and interventional study focusing on the empowerment of families raising children with disabilities who live at home in Japan.

Method

The program consisted of four online peer-based group sessions. Moreover, the families engage in several activities in stages wherein they discover their own issues, find measures to resolve them, and take action, while visualizing interfamily relationships, including social resources, and the status of their family life, with facilitators and other peer members. This study was a non-randomized, waitlist-controlled trial. It compared the results of the intervention group (early group) and the waitlist-controlled group (delayed group). The participants were allocated to the early or delayed group in the order of their applications. The main outcome was family empowerment. Other outcomes are the caregiver burden, awareness of the use of social resources, self-compassion, and the quality of life (QOL) of primary caregivers. The timing of the online surveys was as follows: the initial evaluation (Time 1 [T1]) was conducted before the start of the first early group program, and post-intervention evaluation (Time 2 [T2]) was conducted immediately (within one week) after the early group had completed all four sessions (four weeks) of the program. Follow-up evaluation (Time 3 [T3]) was conducted four weeks after the postintervention evaluation. This timing was the same in the delayed group, but the delayed group attended the program after a four-week waiting period, compared to the early group.

\section{Discussion}

The intention is to evaluate whether the provision of the program developed in this study and the evaluation test design are feasible and to verify the efficacy of this program.

Clinical trial registration

The UMIN Clinical Trials Registry (UMIN000044172), registration date: May 19, 2021. https://upload.umin.ac.jp/cgi-open-bin/ctr/ctr_view.cgi?recptno=R000050422

\section{Background}

With highly advanced medical treatment enabling lifesaving techniques and shortened hospital stays, there are an increasing number of children with severe disabilities who are cared for at home, from childhood. In Japan, there has been a rapid increase in the number of children with severe disabilities who need constant medical care. According to the Ministry of Health, Labor and Welfare, the number of 
children with severe disabilities aged 0-19 years who need constant medical care was 9,403 in 2005, and this number has doubled to 20,155 in 2020 [1].

Families raising children with disabilities face much higher risks to their own health and lives, compared to families raising healthy children. The mother, who is usually the primary caregiver, carries the burden of the time and responsibility associated with providing the required care to the child, and thus tends to have poor mental health [2]. Thus, there is an urgent need for direct support for families raising children with disabilities.

The concept of empowerment began to be used in the field of health sociology since the 1980s in the US and from the 1990s in the UK $[3,4]$. Family empowerment is the ability of families to control their own lives and promote the collaborative raising of children with disabilities [5]. Koren et al. proposed a conceptual framework of empowerment for families raising children with disabilities in the community ("empowerment of family (internal) relationships," "empowerment of relationships with service systems," and "empowerment of interactions with the community") [6]. In Japan, the concept of empowerment was introduced in 1999 in the Guidelines for Support Services, including Care for People with Disabilities [7], and the concept of "family as a whole" for children with disabilities and their families was presented.

We previously conducted in-depth interviews with 34 families with children with severe disabilities who lived at home (mother, father, and siblings aged 12 years and older) [8], and conducted a questionnaire survey of 158 nurses and government officials using the Delphi method [9] to comprehensively list the factors that comprise the family empowerment model. Thereafter, we issued a self-administered questionnaire based on that model to 1,659 families nationwide with children with severe disabilities who were living at home, to develop and verify the family empowerment model [10]. This research showed that family empowerment is high when families recognize that they can "use social resources" and they can take the initiative to reduce their "caregiver burden." However, the above were observational studies. In this study, we verify, through implementational research, whether an intervention in each of its factors (specifically, by working on increasing the "awareness of the use of social resources", and reducing the "caregiver burden" can actually increase family empowerment and determine which of its three levels ("family (internal) FA", "service system SS" and "community SP") are actually empowered by this intervention.

\section{Purpose}

The purpose of this study was to develop an intervention program to promote family empowerment that focuses on multiple factors related to family empowerment, by mainly examining the "awareness of the use of social resources" and the "caregiver burden," which were identified from the results of previous research, and thereafter, verifying the effect of the program. The specific purpose was to evaluate whether participating in this program improves family empowerment. This is the first online program development and interventional study focusing on the empowerment of families raising children with disabilities, who live at home in Japan. 


\section{Method}

\section{Study design and setting}

This study is a non-randomized, waitlist-controlled trial. It compared the group starting the family empowerment program the week after the initial evaluation (intervention /early group) to the group starting delayed intervention (waitlist-controlled/delayed group). This program began in Japan in July 2021, and participants will be registered from April to December 2021. This study is registered as a clinical trial in the UMIN Clinical Trials Registry (UMIN000044172). This protocol has been prepared in accordance with the Standard Protocol Items: Recommendations for Intervention trials (SPIRIT) 2013 statement [11]. The trial and flow of participants through the trial are illustrated in the flow chart (Fig. 1). The SPIRIT schedule of enrolment, interventions, and assessments is presented in Fig. 2. The SPIRIT Checklist can be found in Additional File 1. This protocol is version 1, dated June 2021.

\section{Recruitment method}

A written request for cooperation in the study was sent by post to people in charge of medical institutions, government agencies, and special-needs schools nationwide. The organizations requested to cooperate were specialized medical institutions and rehabilitation centers for children with physical disabilities, university hospitals and specialist pediatric hospitals, medical welfare departments of local governments, and special-needs schools for children with physical impairment or chronic diseases. A total of 20,000 copies of the documents explaining the research were distributed.

People in charge of organizations who consented to cooperate in the study will display posters to recruit program participants within their facility and will distribute the handout explaining the research to people who match the inclusion criteria and do not fall under the exclusion criteria. The distributed handout describes the general outline of the family empowerment program, the schedule, target participants, and implementation method. People who are interested in the study can then access a dedicated website from the information on the handout and apply to participate in the study after consenting to the content of the study's handout. To reach the target sample size, requests for study cooperation may also be sent to associations for families with disabled children and day care centers for children with disabilities, depending on the status of participant registration.

\section{Inclusion criteria, exclusion criteria}

The participants targeted in this study are family caregivers who are primarily caring for children under the age of 20 with physical, intellectual, or developmental disorders, at home. Exclusion criteria included people who were unable to read or write in Japanese, and those who were unable to connect to the internet.

\section{Calculating sample size}


The sample size was estimated by selecting F-tests (ANCOVA) using G*Power software version 3.1.9.7 [12]. The required sample size was calculated based on data obtained from the pretest implemented with the same subjects and equivalent program content used in this study. The required sample size was 52 , assuming an effect size of 0.4 , a test power of $80 \%$, and an a value of 0.05 . The dropout rate was assumed to be $13 \%$; therefore, 30 subjects were assigned to each group, with a total of 60 subjects in both groups.

\section{Intervention allocation}

Participants were allocated to the early or delayed group in the order of their applications. A maximum of 20 people can participate per program, and the family empowerment program comprising a total of four group sessions is implemented several times per month. After allocation to the intervention, the participants are blinded until the follow-up evaluation, by not informing participants in each group of the time of intervention and evaluation in the other group. Facilitators of the family empowerment program could not be blinded to the participants' allocations because they need to contact the participants and respond to inquiries. The analysts are blinded to the allocation of participants.

\section{Preventing participant dropout}

Participants can contact the principal investigator directly by email or telephone with questions about participation in the study prior to participating in the program. In addition, after deciding to participate, participants received individual emails about confirmation of the arrival of the textbook, etc., and guidance on participation methods before the start date of the program. Each participant will also be sent reminders to respond to questionnaires required for evaluation of the program, depending on the available response timeframe and response status of each participant.

\section{Ethical considerations}

The protocol for this study will be reviewed and approved by the institutional review board established by the principal investigator's affiliated institution. Voluntary informed consent will be obtained from the study subjects in writing. The explanatory document for this study includes matters that need to be explained to study subjects according to the "Ethical Guidelines for Life Science and Medical Research Involving Human Subjects" formulated by the Ministry of Health, Labor and Welfare. That is, it includes information on the purpose and significance of the study, the study methods, the burden on the study subjects, expected risks and benefits to the subjects, management of personal information, methods to store and dispose of information, and financial burden on the study subjects. The study subjects may freely choose to participate or abstain from the study without being disadvantaged in any way. Their declaration of intent to participate in the study may be withdrawn at any time without providing a reason. Moreover, participants' personal information and data remain confidential. The data collected in this study, including the questionnaire responses, will be stored for a certain period even after the end of the study.

\section{Intervention methods}


The intervention program for this study was developed using an intervention mapping approach [13]. We developed a peer group-based support program based on social cognitive theory [14] and a taxonomy of behavior change techniques (BCTs), and a practical behavior change technique [15], as an intervention method to encourage changes in family behavior, with the aim of improving family empowerment in families raising children with disabilities who live at home. The specific development process for the intervention used in this study is described in a separate paper (currently under review) [16].

The program held a total of four group sessions every Saturday using an online meeting system $\left(Z O O M{ }^{\circledR}\right)$. The participants received a textbook by post approximately one week before the first program. Four to six researchers took turns as the program facilitator each time. The main facilitator was fixed for all four sessions, while three to four facilitators were responsible for encouraging sharing among participants in breakout rooms and following up with participants about the online meeting system.

The program details are as follows. In the first session, each participant created and shared an eco-map [16] with the aim of "understanding the current circumstances surrounding the child and the family" and the participants reflected upon their own lives and the lives of their disabled children and their families at the present time. Finding elements of family empowerment in each participant's life led to a basic understanding of family empowerment. Furthermore, participants sharing their eco-maps promoted mutual understanding among them and were expected to have the secondary effect of promoting group dynamics. In the second session, all participants shared their homework, which was to create a table to record a week in their lives between the first and second sessions, with the aim of "reflecting on the child's and the family's actual life to clarify the participant's desired life." Time was set aside for participants to think about problems in their current lives and to imagine the kind of life they want for themselves and their families. In the third session, participants engaged in a clear goal setting exercise to achieve the kind of life they want for themselves and for their families, set specific behavior goals to achieve that end and shared this information with the group with the aim of "establishing goals to reach the life they want for themselves and for their family." Time was set aside to think in relation to each domain of family empowerment, which links "What can be done within the family? (FA)," "What can be done with service providers (SS)," and "What can be done with government officials in the local community? (SP)." In the fourth session, participants shared their "efforts to work toward the life they want," which was the homework between the third and fourth sessions and reflected on the group work as a whole.

Throughout the program described above, participants were reminded to set clear goals based on the characteristics of their families, on raising a child with disabilities, to promote self-monitoring, to provide information, to clarify barriers to benefits and risks related to health and life, to promote peer support, to promote self-compassion, and to promote awareness of family empowerment. All the participants gathered together at the start and at the end of the program, and they were separated into two to four groups (around three to six people per group) of online smaller rooms (breakout rooms) in line with the type of disability and the age of the child, during the time for participants to share their opinions and questions and answers. Interactions between parents and children at different developmental stages 
aimed to provide the opportunity for parents with younger children to acquire information on their outlook for the future, and for parents with older children to self-monitor by looking back on their progress to date.

\section{Personal education tools}

We prepared three types of tools: a workbook, a booklet on family empowerment as a sub-textbook, and an online meeting system manual. The textbook, booklet, and online meeting system manual were distributed to the program participants. The online meeting system manual was distributed so that it could be viewed online. The textbook was printed in color, and innovations were added, such as large font and large sheets of paper for homework, to ensure that the participants could comfortably participate in the program. A certificate of completion was inserted at the end of the textbook, and after participating in the last session, a PDF copy of the certificate of completion was emailed to participants, with the aim of improving self-compassion. The booklet was used as a tool to promote awareness of family empowerment. An explanation of the three levels of family empowerment and explanations of each item were provided with illustrations, ensuring that the participants could easily refer to the content during the program and could link the content to family empowerment and their situations.

\section{Facilitators}

The facilitators who oversee the entire program are educators and researchers in pediatrics and family nursing. Furthermore, when the participants were separated into smaller groups, the facilitators in charge of the smaller groups were people with deep ties to the disabled child and the family, such as specialists routinely involved with these children and families, family members, or parties raising these children. A facilitation book was created to standardize the program. This book follows the textbook, describing the time needed for work within the program in detail and points to note to ensure that the quality of progress is sustained among the facilitators.

\section{Data collection method}

The data collected in this study are information reported by the participants via an online survey. A survey request email was sent to the participants regarding the online survey at the times described below, and one or more reminders were sent to people who did not respond. Participants were requested to cooperate in the survey regardless of their program attendance. For example, even if a participant did not attend any sessions, they were asked to complete the survey.

The online survey was conducted in the following three times. After the participants had applied and were allocated to either the early or delayed group, they were notified of their attendance month (from July to December). At the time, participants were not informed if they were in the early or delayed group; they were simply notified of their attendance month to prevent bias in the responses based on the participant's impression of their allocated group. Thereafter, the initial evaluation (Time 1 [T1]) was conducted before the early group started the first program. For the early group, this corresponded roughly to the week before attending the program. The post-intervention evaluation (Time 2 [T2]) was conducted immediately (within 1 week) after the early group completed all four sessions in the program (4 weeks). The follow-up 
evaluation (Time 3 [T3]) was conducted four weeks after the post-intervention evaluation. The timing is the same for the delayed group, but it attends the program precisely four weeks after the early group does. That is, the delayed group completed the evaluation for T1 roughly 5 weeks before attending the program, T2 immediately before attending the program, and T3 immediately after completing the course.

Response information was collected in the online survey system. Participants are allocated identity documents (IDs) that are linked to the response information, and every response of each participant from $\mathrm{T} 1$ to $\mathrm{T} 3 \mathrm{can}$ be linked. If there were multiple responses from the same person at the same time (duplications), it was assumed that it was because the respondent wished to correct their answer; hence, the later response was adopted. The data collected in the online survey system were backed up fortnightly. Once all the participants had completed the follow-up evaluations, the data were downloaded from the system and stored as primary raw data.

\section{Outcomes and measurement methods}

The main outcome was family empowerment. Family empowerment was measured each time at T1-T3 using the Japanese version of the Family Empowerment Scale (FES) [18]. FES evaluates whether a family is able to control its family life while collaborating with others, addressing the three levels of "Family (internal) relationships (FA)," "Relationships with service systems (SS)," and "Involvement with community (SP)." Answers to 34 items on a 5-point scale were added up, and the results were scored with the total score and three subscale scores. If some responses were missing, but other items were answered, the missing data were supplemented by averaging out the answered items. The higher the score, the higher the level of family empowerment.

Other outcomes are the caregiver burden, awareness of the use of social resources, self-compassion, and the QOL of primary caregivers. The caregiver burden was measured using the short form of the Japanese version of the Zarit Caregiver Burden Interview (J-ZBI-8) [19]. The ZBI-8 evaluates caregiver burden, including physical and mental burden and constraints on social activities. Eight items were ranked on a 5point scale, and the caregiver burden score was obtained as the total score by summing the responses. If some responses were missing, but other items were answered, the missing data were supplemented by averaging out the answered items. The higher the score, the greater the caregiver burden. These questions were asked each time at T1-T3.

It has been shown that parents raising children with disabilities have sleep disturbances, which creates a burden for parents [20]. Therefore, we added two items on sleep-related matters. One question inquires whether the parent gets up during the night to care for the child: "How often do you need to get up during the night to care for your child with disabilities?" The respondents chose answers from "Every night," "Several nights a week," "Several nights a month," "Never," and "Other (state specific information)". The other question asked about the average hours of sleep per day. These items were asked each time at T1T3. 
Two original items were created regarding the use of social resources based on discussions among the researchers in our previous study [10]. The first question is asked each time at T1-T3: "Do you feel you can use social resources, properly?" and respondents can select from "I often use social resources," "I sometimes use social resources," "I do not use social resources very often," and "I never use social resources." The other question asked at T2 and T3 is: "Do you feel that you are now more able to properly use social resources compared to when you answered the previous questionnaire?" and the respondents selected "Can no longer use social resources," "Cannot use social resources very much," "Unchanged," "Can use social resources slightly more," or "Can use social resources very often." The survey also inquired about the participants' actual use of social resources (actual use volume and frequency) as basic attributes.

Self-compassion was measured each time at T1-T3 using the short form of the Japanese version of the Self-Compassion Scale (SCS) [21]. SCS is comprised of 12 items on a 5-point scale, and there are two items applicable to each of the following aspects of "self-kindness," "self-judgement," "common humanity," "isolation," "mindfulness," and "over-identification," and six scaled scores are obtained by totaling the answers. If a response is missing, but the other item in the same aspect has been answered, the missing data are supplemented by the answered item. The higher the score, the higher the level of self-compassion.

The QOL of the primary caregiver was measured each time at T1-T3 using the SF-8 [22]. SF8 is an 8item, 5-6-point scale, and the score is calculated based on the distribution of 50 points as the standard value using a unique scoring algorithm. Missing data are also handled using this scoring algorithm. The higher the score, the higher the health-related QOL.

In addition to these outcomes, the following questions were asked about basic attributes. At T1, the participants were asked about their relationship (from the child's perspective), age (in 10-year increments), marital status, cohabitation status with partner, highest level of education, occupation, household income, and family members living in the same household. At T1, the following questions were asked about the child's attributes: gender, age, name of diagnosis, age at diagnosis, school attendance history, condition of disability, and required care. At T2 and T3, the respondents were asked if there were any changes in these circumstances and were asked to provide details if there were any. To ensure that the responses were not compulsory, the respondents were given the option of, "No response" and the online survey system was arranged so that respondents could progress to the next page without answering some or all questions.

The respondents were asked the following questions in the questionnaire to evaluate the process after attending the program and four weeks later: How did you feel after attending the program? Did you tell your family about the content of the program and the information you learned? Would you recommend the program to your friends? How can the program be expanded? They were asked their opinion about the program (age of their child when they attended the program, number of sessions, days the sessions were held, the length of the program, and the necessity of childcare). 


\section{Data analysis}

This program is designed so that attending a total of four sessions once fortnightly will enable participants to sequentially review their family situation including the relationships within their own families and their social resources, their own and their family members' lives, and to visualize their family relationships, burden, and the use of services. Specifically, it is expected that participants will have an improved sense of family empowerment immediately after attending the program, including establishing and practicing specific and feasible plans. Therefore, the primary outcome of this program was the total FES score at T2 to verify whether the program was effective. The secondary outcomes were to verify improvement in levels of family empowerment (FES subscale score at T2), whether aspects directly worked on in the program had improved (caregiver burden, use (awareness) of social resources, selfcompassion at T2), and whether they had a comprehensive effect (health-related QOL at T2).

Furthermore, whether any interventional effect observed at T2 was sustained, was verified (each outcome score at T3 for the early group), and whether any interventional effect observed in the early group was reproduced (each outcome score at T3 for the delayed group). The statistical analysis plan is described as follows:

The responses will be used where the outcome scores can be calculated as valid responses, and ITT analysis will be performed to manage data from participants who had deviated from the study protocol by not attending the program at the designated times as the original allocation group. First, descriptive statistics were calculated for the background attributes and outcome scores of participants (both the participant and the child) at T1. The interval scale was used to calculate the mean and standard deviation; ordinal and nominal scales were used to calculate the frequency and proportion, and intergroup comparison was performed using Welch's t test, the Mann-Whitney $U$ test, and Fisher's exact probability test.

There may be participants who drop out between T1 and T2 and between T2 and T3. We compared the background attributes and outcome scores at T1 between the primary outcomes "total FES score of valid respondents at T2" and "non-respondents at T2 (but were valid respondents at T1)" to examine and discuss the effect caused by participants dropping out. In addition, an intergroup comparison of background attributes and outcome scores at T1 will be conducted for a population of only a "total FES score of valid respondents at T2." If group differences that may create significant bias or biased dropouts have been observed in the analysis to date, we will consider adding those variables as covariates in subsequent analyses.

The primary analysis is an intergroup comparison using analysis of covariance with the total FES score at $\mathrm{T} 1$ as the covariate, and the total FES score at $\mathrm{T} 2$ as the dependent variable. If there was a significant interaction between the independent variable (group: early group or delayed group) and the covariate (total FES score at T1), intergroup comparison will be conducted using ANOVA without considering the scores at T1. The level of statistical significance was set as $5 \%$. Interim analysis will not be performed. Subgroup analysis will be conducted for children with severe disabilities, children with disabilities who 
need constant medical care, and single-parent households. We will also examine whether the increase in the total FES score from T1 to T2 is clinically significant. However, the clinical minimum significant difference for FES is not clear, so this will be discussed through a comparison with a previous study [10].

An intergroup comparison will be similarly conducted for the secondary outcomes using analysis of covariance for each outcome at $\mathrm{T} 2$. The results at $\mathrm{T} 1$ and $\mathrm{T} 3$ for the early group will be examined using a paired t-test to determine whether the intervention effect observed at T2 is sustained. The results at T2 and T3 for the delayed group will be examined using a paired t-test to determine whether the intervention effect observed in the early group is reproduced in the delayed group.

The following two analyses will be conducted as a sensitivity analysis of the primary analysis. The first is a re-analysis of the dropouts from T1 to T2 with the amount of change in the total FES score set as 0 , to consider the effect of dropouts. The second is a per-protocol analysis. That is, re-analysis will be conducted to compare the results of participants who attended all four sessions of the program (group) to all others (group).

\section{Data Management}

All self-reported data will be entered directly into a web-based data capture system via an online survey.

Only the all researchers on the team will have access to the data set, and the data will be password protected.

This study is low-risk, we think that a data monitoring committee was not necessary.

\section{Discussion}

We developed a peer-group based online intervention program with the aim of empowering families to raise children with disabilities. There are few evidence-based programs in research on intervention programs for families with children with disabilities [23,24]. This is the first initiative for an evidencebased program for families raising children with disabilities at home that has been developed, implemented, and scientifically evaluated in Japan. This study provides a detailed protocol for the intervention program.

The family empowerment program we developed for families with children with disabilities uses a participant-led approach, enabling participants to discover their own problems, and find and action measures to resolve those problems through activities, while visualizing interfamily relationships, including social resources, and the current situation of family life. These peer group-based activities are performed in stages, and the expected outcomes for the participating families are improvements such as improved empowerment, reduced caregiver burden, and improved utilization of social resources. An expected secondary effect of participation in a peer group-based program is that it may lead to the formation of social networks for these families. 
Parents of children with disabilities are always busy raising and caring for these children and their siblings and are unable to get enough sleep or free time for themselves. Therefore, it is difficult for these people to participate in intervention programs. However, this time, improvements were made to the content of the program so that it could be conducted online during the coronavirus disease 2019 (COVID19) crisis. Consequently, this approach allowed families to connect with other families located far away who were also raising children with disabilities, without leaving home. The participants were able to share their experience of discovering their own problems and to find measures to resolve those problems, which increased the feasibility of the program.

Converting the results of this study to an evidence-based community program and widely disseminating the program will be a challenge. The results of this study promote the empowerment of families with children with disabilities and may contribute to improve the health of these families. In the future, it will be necessary to verify the effect of this program and to determine if there are any adverse effects by running pilot tests. It is also essential to improve the program based on the results of the verification.

\section{Abbreviations}

BCT: behavior change technique; COVID-19: coronavirus disease 2019; FA: family (internal); FES: Family Empowerment Scale; ID: identity document; QOL: quality of life; SCS: Self-Compassion Scale; SP: community; SS: service system; T1: Time 1; T2: Time 2; T3: Time 3;-ZBI-8: Zarit caregiver burden interview

\section{Declarations}

\section{Acknowledgements}

We would like to thank all of the mothers of children with disabilities who were involved in the development of the interventions for this study. We would like to thank Editage (www.editage.jp) for English language editing.

\section{Authors' contributions}

$\mathrm{RW}$ is principal investigator, conceived of the trial and led the trial design, overall analysis plan and funding application. AM, RW, IS, FH, KN, and SS drafted the manuscript, and NI provided revisions to the clinical content. RW, AM and NK led the development the initial program content of the program. IS manage the trial and data collection. IS and SS developed the statistical analysis plan. All authors contributed to the design of the trial and its procedures. All authors read and approved the final manuscript.

\section{Funding}

This research was supported by JSPS KAKENHI $18 \mathrm{H} 03093$ (Grant-in-Aid for Scientific Research B).

\section{Declarations}

Page $12 / 16$ 


\section{Ethics approval and consent to participate}

Ethics approval for the study was received on 22 August 2019 from the University of Tsukuba Medical Research Ethics Committee (Approval Number 1420); recruitment is currently ongoing.

\section{Consent for publication}

Not applicable; no study data are included in this publication.

\section{Availability of data and materials}

Not applicable.

\section{Competing interests}

The authors declare that they have no competing interests.

\section{References}

1. Ministry of Health, Labor and Welfare website: Support measures for severely handicapped children who need constant medical care and their families https://www.mhlw.go.jp/stf/ seisakunitsuite/bunya/hukushi_kaigo/shougaishahukushi/service/index_00004.html (Access date: 2021.09.12)

2. Yamaoka Y, Tamiya N, Moriyama Y, Sandoval Garrido FA, Sumazaki R, Noguchi H. Mental health of parents as caregivers of children with disabilities: Based on Japanese nationwide survey. PLOS ONE. 2015;10(12):e0145200. doi: 10.1371/journal.pone.0145200

3. Oda K, Sugimoto T, Hisada N, (editors). Theories and techniques of empowerment practice. Tokyo: Chuohoki Publishing Co, Ltd; 1999

4. Ogawa Y. Empowerment of persons with disabilities - Welfare for persons with disabilities in the UK. 167-168. Tokyo: Akashi Shoten; 1998

5. Segal SP, Silverman C, Temkin T. Measuring empowerment in client-run self-help agencies. Community Ment Health J. 1995;31:215-27

6. Koren PE, DeChillo N, Friesen BJ. Measuring empowerment in families whose children have emotional disabilities: A brief questionnaire. Rehabil Psychol. 1992;37:305-21

7. Planning Division, Department of Health and Welfare for Persons with Disabilities, Minister's Secretariat $\triangle$ Training text for care managers of persons with. Disabilities. 431. 1999. Tokyo: Chuohoki Publishing Co, Ltd 
8. Wakimizu R, Fujioka H, Numaguchi C, Nishigaki K, Sato N, Yamaguchi K. Own role recognized by mothers, fathers and siblings living with children with severe motor and intellectual disabilities, expected roles of other family members and support needs as a family. Int Nurs Care Res. 2015;14(4):1-10

9. Wakimizu R, Fujioka H, Numaguchi C, Nishigaki K, Sato N, Yamaguchi K. Support needs of families living with children with severe motor and intellectual disabilities and evaluation of importance and practicality by specialists-From a Delphi method-based survey of nurses and government officials -. Journal of Health and Welfare Statistics. 2016;63(4):23-32

10. Wakimizu R, Fujioka H, Nishigaki K, Matsuzawa A, Iwata N, Kishino M et al. Construction of empirical model focused on the family empowerment nurturing the child with severe motor and intellectual disabilities at home in Japan. J Child Health. 2018;77(5):423-32

11. Chan W, Terzlaff JM, Altman DG, Laupacis A, Gøtzsche PC, Krleža-Jerić K, et al. SPIRIT 2013 statement: defining standard protocol items for clinical trials. Ann Intern Med. 2013; 158(3):200-7. doi: 10.7326/0003-4819-158-3-201302050-00583.

12. Faul F, Erdfelder E, Lang AG, Buchner A. G* Power 3: A flexible statistical power analysis program for the social, behavioral, and biomedical sciences. Behav Res Methods. 2007;39(2):175-91

13. Bartholomew LK, Parcel GS, Kok G, Gottlieb NH, Fernandez ME. Planning health promotion programs: An intervention mapping approach. 4th ed. San Francisco: Jossey-Bass; 2016

14. Bandura A. Social foundations of thought in action: A social cognitive theory. Englewood Cliffs, NJ: Prentice Hall; 1986

15. Abraham C, Michie S. A taxonomy of behavior change techniques used in interventions. Health Psychol. 2008;27(3):379-87. doi:10.1037/0278-6133.27.3.379

16. Wakimizu R, Fujioka H, Nishigaki K, Sato I, Iwata N, Matsuzawa A. Development of family empowerment programs for caregivers of children with disabilities at home: Interim report up to "implementation of pretesting". J Int Nurs Res. 2021

17. Ray RA, Street AF. Ecomapping: An innovative research tool for nurses. J Adv Nurs. 2005;50(5):545-52. DOI: 10.1111/j.1365-2648.2005.03434.x

18. Wakimizu R, Fujioka H, Furuya K, lejima A, Yoneyama A. Development of the Japanese Version of the Family Empowerment Scale (J-FES). J Health Welf Stat. 2010;57:33-41

19. Arai Y, Tamiya N, Yano E. The short version of the Japanese version of the Zarit Caregiver Burden Interview (J-ZBI_8): Its reliability and validity. Nihon Ronen Igakkai Zasshi. 2003;40(5):497-503

20. Keilty K, Cohen E, Ho M, Spalding K, Stremler R. Sleep disturbance in family caregivers of children who depend on medical technology: A systematic review. J Pediatr Rehabil Med. 2015;8(2):113-30. 
21. Arimitsu K, Aoki Y, Furukita M, Tada A, Togashi R. Construction and validation of a short form of the Japanese version of the Self-Compassion Scale. Komazawa Annu Rep Psychol. 2016;18:1-9

22. Fukuhara S, Suzukamo Y. Health related QOL scale-SF-8 and SF-36. J Exp Med. 2005;213:133-6

23. Borek AJ, McDonald B, Fredlund M, Bjornstad G, Logan S, Morris C. Healthy Parent Carers programme: development and feasibility of a novel group-based health-promotion intervention. BMC Public Health. 2018;18(1):270. doi: 10.1186/s12889-018-5168-4

24. Bourke-Taylor HM, Joyce KS, Grzegorczyn S, Tirlea L. Mental Health and Health Behaviour Changes for Mothers of Children with a Disability: Effectiveness of a Health and Wellbeing Workshop. J Autism Dev Disord. 2021; 16:1-14. doi: 10.1007/s10803-021-04956-3.

\section{Figures}

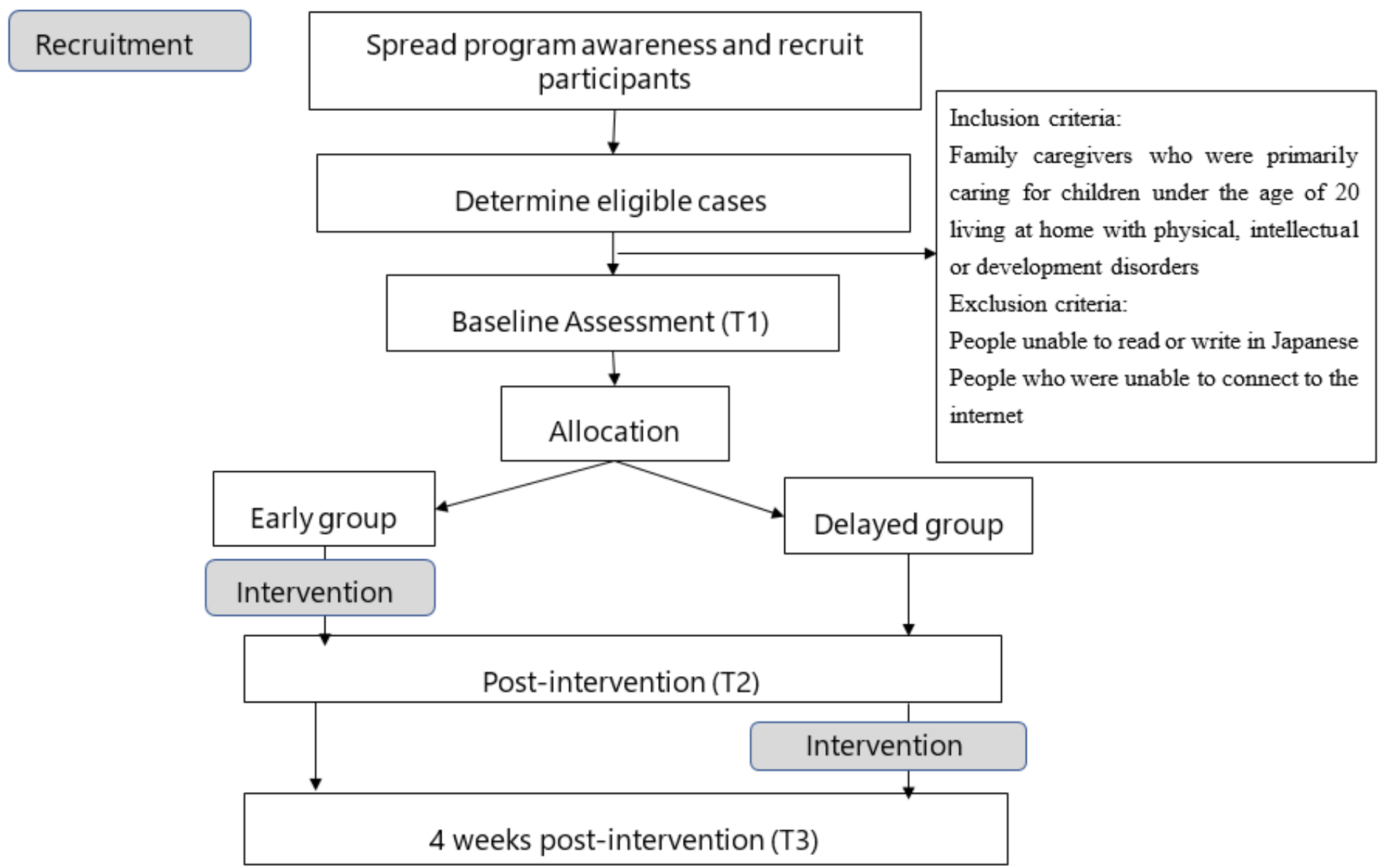

Analysis

\section{Figure 1}


Trial flow chart, $\mathrm{T} 1=$ time $1 ; \mathrm{T} 2=$ time $2 ; \mathrm{T} 3=$ time 3

\begin{tabular}{|c|c|c|c|c|}
\hline \multirow[b]{3}{*}{ TIMEPOINT(T) } & \multicolumn{4}{|c|}{ STUDY PERIOD } \\
\hline & \multirow{2}{*}{$\begin{array}{c}\text { Enrolment } \\
T_{1}\end{array}$} & \multirow{2}{*}{$\begin{array}{c}\text { Allocation } \\
0\end{array}$} & \multicolumn{2}{|c|}{ Post-allocation } \\
\hline & & & $\begin{array}{c}\text { Post- } \\
\text { Intervention } \\
T_{2}\end{array}$ & $\begin{array}{c}4 \text { weeks } \\
\text { Post- } \\
\text { intervention } \\
T_{3} \\
\end{array}$ \\
\hline \multicolumn{5}{|l|}{ ENROLMENT: } \\
\hline \multirow{2}{*}{$\begin{array}{l}\text { Eligibility screen } \\
\text { Informed consent }\end{array}$} & $X$ & & & \\
\hline & $\mathrm{X}$ & & & \\
\hline Allocation & & $\mathrm{X}$ & & \\
\hline \multicolumn{5}{|l|}{ INTERVENTIONS: } \\
\hline \multicolumn{5}{|l|}{$\begin{array}{r}\text { Intervention group } \\
\text { /Early group }\end{array}$} \\
\hline \multicolumn{5}{|l|}{$\begin{array}{r}\text { Waitlist-controlled groups } \\
\text { /Delayed group }\end{array}$} \\
\hline \multicolumn{5}{|l|}{ ASSESSMENTS: } \\
\hline \multirow{2}{*}{$\begin{array}{r}\text { Demographics of } \\
\text { participants and their } \\
\text { children and families } \\
\text { Short version of the Zarit } \\
\text { Caregiver Burden Interview } \\
(\mathrm{J}-\mathrm{ZBI} 8) \\
(8 \text { items) }\end{array}$} & $\mathrm{X}$ & & $\mathrm{X}$ & $\mathrm{X}$ \\
\hline & $X$ & & $X$ & $X$ \\
\hline \multirow{2}{*}{$\begin{array}{r}\text { Awareness of the use of } \\
\text { social resources } \\
\text { Short form of the Japanese } \\
\text { version of the Self- } \\
\text { Compassion Scale (SCS-12) } \\
\text { (12 items) } \\
Q O L \text { of primary careqiver }\end{array}$} & $\mathrm{X}$ & & $\mathrm{X}$ & $\mathrm{X}$ \\
\hline & $\mathrm{X}$ & & $\mathrm{X}$ & $\mathrm{X}$ \\
\hline \multirow{2}{*}{$\begin{array}{r}\text { (SF-8) } \\
(8 \text { items) } \\
\text { Japanese version of the } \\
\text { Family Empowerment Scale } \\
(\mathrm{J}-\mathrm{FES}) \\
\text { (34 items) }\end{array}$} & $\mathrm{X}$ & & $\mathrm{X}$ & $\mathrm{X}$ \\
\hline & $\mathrm{X}$ & & $\mathrm{X}$ & $\mathrm{X}$ \\
\hline Feedback of program & & & $\mathrm{X}$ & $\mathrm{X}$ \\
\hline
\end{tabular}

\section{Figure 2}

The SPIRIT schedule of enrolment, interventions, and assessments

\section{Supplementary Files}

This is a list of supplementary files associated with this preprint. Click to download.

- Additionalfile.1SPIRITFillablechecklist20211019.doc 